\title{
Asociación entre síntomas depresivos del cuidador principal y problemas conductuales en una muestra de preescolares chilenos de 30 a 48 meses de edad Association between primary caregiver depressive symptoms and behavioral problems in a sample of chilean preschool children age 30 to 48 months
}

\author{
Danisa Torres M. ${ }^{1, a}$, Paola Viviani G. ${ }^{2, b}$, Elisa Cohelo M. ${ }^{1}$ y Paula Bedregal G. ${ }^{2}$
}

\begin{abstract}
Introduction: The mental health of the caregiver influences the development of children; however we do not know if there is an association between depressive symptoms of the caregiver and behavioral problems in Chilean preschoolers. The objective was to analyze the association between primary caregiver depressive symptoms and behavioral problems in preschoolers users of the Chilean public health network. Method: Cross-sectional study. Two-stage stratified cluster sampling. Sample 1377 preschool children 2,5-4 years old and their primary caregivers. In order to assess child behavior, a sociodemographic survey and Child Behavior Checklist $1 / 2-5$ we applied to each caregiver, under informed consent. Caregiver depressive symptoms were assessed using the International Diagnostic Composite Interview Short Form. Results: Preschool age was $40.95 \pm 4.6$ months, $51.3 \%$ were boys. 97.5\% of caregivers were female, (ages $31.6 \pm 10.2 \mathrm{yr}$, average schooling 9.5 years). Behavioral problems of clinical rank were found in the three scales: total behavioral problems $21.4 \%$, internalizing $23.1 \%$, and externalizing behaviors $16.4 \% .9 .1 \%$ of caregivers experienced depressive symptomatology. In preschool children with internalizing behavioral, caregivers showed depressive symptoms in 14,5\% of cases, and 16,1\% in those with an externalizing behavioral. In multinomial logistic regression analysis adjusted for confounding variables, bivariate association for internalizing behavioral problems was lost, but persisted for total behavioral problems and for externalizing behavioral. Conclusions: The association between caregiver's depressive symptoms and behavioral problems in Chilean preschool children is consistent and modulated by other medical and psychosocial variables, suggesting the importance of identification, prevention and early intervention of the dyad and their environment.
\end{abstract}

Key words: Preschool Child, Caregiver depression, CBCL.

Rev Chil Neuro-Psiquiat 2018; 56 (2): 100-109

Recibido: 7/09/2017

Aceptado: 5/06/2018

Los autores no presentan ningún tipo de conflicto de interés.

Fuente de apoyo financiero: El estudio se basó en la información disponible en estudio "Levantamiento y análisis de información sobre desarrollo infantil y sus principales determinantes sociales y económicos de niños y niñas que participaron del Programa de Apoyo al Desarrollo Biopsicosocial del Programa Chile Crece Contigo" en 2013, que contó con financiamiento público del Ministerio de Desarrollo Social. No tuvo influencia en el diseño del estudio, ni en la recolección, análisis o interpretación de los datos, ni en la preparación, revisión o aprobación del manuscrito.

Departamento de Psiquiatría, Escuela de Medicina, Pontificia Universidad Católica de Chile.

Departamento de Salud Pública, Escuela de Medicina, Pontificia Universidad Católica de Chile.

Residente del Programa de Psiquiatría del Niño y del Adolescente, Pontificia Universidad Católica de Chile.

Doctor en Estadística. 


\section{Introducción}

A ctualmente no existe consenso sobre los criterios que definen la mayoría de los trastornos psiquiátricos y del desarrollo en preescolares. Los sistemas existentes no ofrecen hasta ahora una clasificación completa y reconocida para su diagnóstico $^{1,2}$. La conducta social, emocional y conductual típica del preescolar se confunde con la patológica ya que la línea que las separa queda definida por la intensidad sintomatológica. Esto ha sido poco estudiado, mostrando distintas prevalencias/frecuencias.

Para distinguir el comportamiento típico de los niveles que merecen atención clínica se necesitan muestras poblacionales. Para ello el Child Behavior Checklist 11/2-5 años (CBCL-11/2-5) 3 se adapta bien a las investigaciones a gran escala porque es barato, fácil de administrar y produce resultados cuantitativos. Sus autores plantean el comportamiento como la manifestación de conductas, emociones y aspectos sociales que pueden estudiarse mediante 3 escalas: total de problemas conductuales (TPC), conductas internalizantes (CI) y conductas externalizantes $(\mathrm{CE})^{4}$.

Los problemas conductuales surgen en los primeros años de vida, están asociados con trastornos de salud mental a largo plazo y sin intervención pueden persistir ${ }^{5}$. Los problemas emocionales y conductuales son los motivos de consulta más frecuentes en salud mental en primera infancia y en preescolares ${ }^{1,6} y$ son un factor de riesgo para desarrollar problemas de salud mental más graves ${ }^{7}$ por lo que es importante su detección temprana. Estos aparecen antes de los 2 años de edad ${ }^{1}$, son estables en el tiempo ${ }^{6}$, la recurrencia de comorbilidad entre problemas internalizantes y externalizantes lleva a policonsulta ${ }^{5,8}$ y la detección temprana permite intervención pronta efectiva9 9

Los procesos biológicos, psicológicos y sociales están implicados en la etiología a través de efectos acumulativos e interactivos ${ }^{10}$, los cuales deben analizarse de forma integrada para poder explicar cuándo en un preescolar estamos frente a un problema conductual. En la evaluación de la conducta del preescolar hay tres aspectos del desarrollo y del entorno importantes de considerar: cambios físicos y cognitivos con emociones complejas ${ }^{11}$, psicopatología del cuidador principal ${ }^{12} \mathrm{y}$, temperamento $\mathrm{y}$ desarrollo del carácter ${ }^{13}$.

La depresión del cuidador principal, especialmente materna, tiene relación con problemas conductuales en preescolares predisponiendo a psicopatología internalizante y externalizante futura $^{14-16}$. La depresión materna genera inadecuada adaptación en los niños ${ }^{17,18}$ que se refleja en múltiples dimensiones del funcionamiento conductual, socio-emocional y familiar ${ }^{18}$. Otras investigaciones han encontrado asociaciones consistentes entre la depresión materna y/o del cuidador y las interrupciones en el funcionamiento socio-emocional e instrumental en todo el curso del desarrollo ${ }^{19,20}$.

Seguel y colaboradores, con otro instrumento, identificaron un 29,7\% de los preescolares de Santiago con alteraciones conductuales y socioemocionales relacionadas con factores psicosociales, como el nivel socioeconómico (NSE) y, además, correlacionaron el estado anímico de la madre con comportamientos ansiosos en sus hijos ${ }^{21}$.

El objetivo de este estudio fue analizar la asociación entre síntomas depresivos del cuidador principal y problemas conductuales en preescolares chilenos. Conocer esta información puede contribuir a orientar intervenciones con enfoque promocional, preventivo en salud mental temprana individual y diádica, y a enfocar recursos para manejo precoz de las conductas desadaptativas persistentes que han demostrado ser estables y predisponentes a psicopatología futura.

\section{Material y Método}

\section{Población y procedimiento}

La población estuvo constituida por niños y niñas de 30 a 48 meses de edad y sus cuidadores principales quienes asistían regularmente desde el control prenatal a los centros de atención primaria en el sector público de salud. Se obtuvo una muestra aleatoria, bietápica por conglomerados, representativa nacional $(\mathrm{n}=1.377)$ en la que se registró 
el comportamiento y competencias socioemocionales del niño reportadas por el cuidador principal utilizando CBCL-1 $1 / 2-5^{3}$. En el cuidador principal se pesquisaron síntomas depresivos mediante la Entrevista Diagnóstica Internacional Compuesta abreviada (CIDI-SF) ${ }^{22}$.

Estos datos fueron obtenidos, como parte del levantamiento y análisis de información sobre desarrollo infantil y sus principales determinantes sociales y económicos de niños y niñas que participaron del Programa de Apoyo al Desarrollo Biopsicosocial del Programa Chile Crece Contigo (PChCC) en el año 2013. Mediante la encuesta del PChCC aplicada en hogares, por encuestadores capacitados, se obtuvieron variables sociodemográficas e intermedias de interés donde se identificó y caracterizó, además, al cuidador principal y al preescolar. El levantamiento de la información fue liderado por el Instituto de Sociología de la Pontificia Universidad Católica de Chile, con la supervisión del Departamento de Salud Pública de la misma institución, contó con la aprobación del comité de ética de investigación del Servicio de Salud Metropolitano Oriente y con el consentimiento informado de los cuidadores principales de los niños/as.

\section{Instrumentos}

El CBCL-1 1/2-5 es una entrevista estructurada que evalúa el comportamiento social, emocional y conductual de los 2 últimos meses en niños/as de 18 a 60 meses de edad. Contiene 100 preguntas que contestan los padres o adulto referente, una de ellas abierta, con las que se obtienen el TPC, CI (36 preguntas) y CE (24 preguntas).

Cada pregunta se califica en una escala Likert: $0=$ no es cierto, $1=$ a veces cierto, y $2=$ a menudo es cierto, cuya sumatoria permite obtener un puntaje bruto (Score Total) por pregunta y un puntaje bruto promedio tanto para TPC como para CI y $\mathrm{CE}$ que corresponden a las 3 escalas de evaluación. Mediante un algoritmo de la puntuación se obtiene un puntaje $\mathrm{T}$ (Score $\mathrm{T}$ ) que pondera el problema conductual en un rango normal $(<60)$, borderline (60-63) o rango clínico (>63-100) en cada una de las escalas, las cuales no son mutuamente exclu- yentes y donde TPC no es la sumatoria de las dos anteriores. Su uso es aplicable en estudios epidemiológicos y transculturales, para fines clínicos y de investigación.

Se establecieron puntajes bruto promedio y $\mathrm{T}$ basados en las recomendaciones del Manual del instrumento ${ }^{3}$. La consistencia interna en la muestra fue $>0,7$ para todos los constructos ${ }^{23}$.

El CIDI-SF ${ }^{22}$ es una encuesta estructurada que identifica la presencia y severidad de síntomas depresivos en adultos el año previo. Su uso es aplicable en estudios epidemiológicos y transculturales, para fines clínicos y de investigación. Se registraron los criterios mayores para depresión: tristeza y anhedonia.

\section{Análisis estadístico}

Se realizó con SPSS versión 19.0. La calificación de las TPC, CI y CE se caracterizaron mediante medidas de resumen (promedio y desviación estándar) e intervalos de confianza del 95\% (IC 95\%). La asociación entre síntomas depresivos del cuidador principal y los problemas conductuales se evaluó mediante $\chi^{2}$. Además, se buscó asociación entre diversas exposiciones y el desarrollo de problemas conductuales. Las variables candidatas fueron evaluadas con análisis bivariado mediante chi-cuadrado o ANOVA según corresponda. Las variables que resultaron significativas en este análisis se introdujeron en un modelo multivariado de regresión logística multinomial, con estimación de odds ratios (OR) e IC 95\%. La significación estadística para todas las pruebas se estableció en un valor $\mathrm{p}<0,05$.

\section{Resultados}

Se entrevistaron 1.377 cuidadores principales, 97,5\% fueron mujeres, $86,3 \%$ de ellas correspondieron a la madre, el $8,4 \%$ a la abuela y el 2,8\% a otras. La Tabla 1 expone las características sociodemográficas de la muestra.

El score total promedio para TPC fue $43,21 \pm 28,6$, para CI y CE fue de 12,31 $\pm 10,56$ y $15,44 \pm 9,27$ respectivamente. 
Tabla 1. Características sociodemográficas de niños/as y sus cuidadores

\begin{tabular}{|c|c|c|c|c|c|c|c|c|c|c|}
\hline \multirow[t]{2}{*}{$\begin{array}{l}\text { Características sociodemográficas de } \\
\text { niños/as y sus cuidadores }\end{array}$} & \multicolumn{2}{|c|}{$\begin{array}{c}\text { Total de la } \\
\text { muestra } \\
\mathbf{n}=1.377\end{array}$} & \multicolumn{2}{|c|}{$\begin{array}{c}\text { Sin problemas } \\
\text { conductuales } \\
\mathbf{n}=759\end{array}$} & \multicolumn{2}{|c|}{$\begin{array}{c}\text { TPC } \\
\mathrm{n}=295\end{array}$} & \multicolumn{2}{|c|}{$\begin{array}{c}\text { CI } \\
\mathrm{n}=318\end{array}$} & \multicolumn{2}{|c|}{$\begin{array}{c}\mathrm{CE} \\
\mathrm{n}=226\end{array}$} \\
\hline & $\begin{array}{l}\text { Media/ } \\
\%\end{array}$ & DS & $\begin{array}{l}\text { Media/ } \\
\%\end{array}$ & DS & $\begin{array}{l}\text { Media/ } \\
\%\end{array}$ & DS & $\begin{array}{l}\text { Media/ } \\
\%\end{array}$ & DS & $\begin{array}{l}\text { Media/ } \\
\%\end{array}$ & DS \\
\hline \multicolumn{11}{|l|}{ Del Niño/a } \\
\hline Edad & 40,95 & 4,6 & 41,18 & 4,7 & 40,46 & 4,4 & 40,81 & 4,4 & 40,66 & 4,5 \\
\hline \multicolumn{11}{|l|}{ Sexo } \\
\hline Hombre & 51,3 & & 50,3 & & 57,3 & & 55,3 & & 56,2 & \\
\hline Mujer & 48,7 & & 49,7 & & 42,7 & & 44,7 & & 43,8 & \\
\hline Asiste a jardín infantil & 50 & & 47,8 & & 48,8 & & 50,3 & & 53,1 & \\
\hline $\begin{array}{l}N^{\circ} \text { de enfermedades crónicas en la } \\
\text { infancia }\end{array}$ & 1,1 & 1,3 & 0,88 & 1,2 & 1,5 & 1,5 & 1,5 & 1,5 & 1,4 & 1,4 \\
\hline \multicolumn{11}{|l|}{ Del Cuidador Principal } \\
\hline Edad & 31,6 & 10,2 & 32,4 & 10,5 & 30,7 & 9,7 & 30,8 & 9,9 & 30,5 & 9,0 \\
\hline \multicolumn{11}{|l|}{ Sexo } \\
\hline Hombre & 2,5 & & 3,2 & & 1 & & 1,6 & & 1,8 & \\
\hline Mujer & 97,5 & & 96,8 & & 99 & & 98,4 & & 98,2 & \\
\hline \multicolumn{11}{|l|}{ Estado civil } \\
\hline Divorciado & 14,8 & & 12,5 & & 21,4 & & 22,6 & & 21,7 & \\
\hline No divorciado & 85,2 & & 87,5 & & 78,6 & & 77,4 & & 78,3 & \\
\hline \multicolumn{11}{|l|}{ Empleo } \\
\hline Empleado & 82,2 & & 84,1 & & 76,6 & & 78,3 & & 81,9 & \\
\hline Desempleado & 17,8 & & 15,9 & & 23,4 & & 21,7 & & 18,1 & \\
\hline \multicolumn{11}{|l|}{ Nivel educacional } \\
\hline Sin enseñanza básica & 1,1 & & 0,8 & & 2 & & 2,2 & & 1,3 & \\
\hline Enseñanza básica & 20,4 & & 19,9 & & 23,1 & & 23,3 & & 24,3 & \\
\hline Enseñanza media & 66,7 & & 67 & & 67,5 & & 67,3 & & 65,5 & \\
\hline Estudios superiores & 11,8 & & 12,3 & & 7,5 & & 7,2 & & 8,8 & \\
\hline Años de estudios & 9,5 & 3,6 & 9,4 & 3,6 & 9,3 & 3,5 & 9,3 & 3,5 & 9,5 & 3,6 \\
\hline $\mathrm{N}^{\circ}$ de eventos vitales estresantes & 1,2 & 1,5 & 1,1 & 1,5 & 1,6 & 1,5 & 1,6 & 1,5 & 1,5 & 1,6 \\
\hline \multicolumn{11}{|l|}{$\begin{array}{l}\text { Emocionalmente, atender al niño/a le } \\
\text { demanda }\end{array}$} \\
\hline Mucho esfuerzo & 17,9 & & 10,7 & & 31,0 & & 27,4 & & 36,7 & \\
\hline Moderado esfuerzo & 36,2 & & 37,2 & & 35,0 & & 35,3 & & 35,4 & \\
\hline Escaso esfuerzo & 45,9 & & 52,2 & & 34,0 & & 37,2 & & 27,9 & \\
\hline \multicolumn{11}{|l|}{ Síntomas depresivos del cuidador } \\
\hline Sí & 9,1 & & 6,8 & & 17,0 & & 14,5 & & 16,1 & \\
\hline No & 90,9 & & 93,2 & & 83,0 & & 85,5 & & 83,9 & \\
\hline \multicolumn{11}{|l|}{ Del grupo familiar } \\
\hline \multicolumn{11}{|l|}{ Nivel socioeconómico } \\
\hline Alto & 8,3 & & 9,0 & & 7,5 & & 7,9 & & 8,4 & \\
\hline Medio-Alto & 14,1 & & 14,2 & & 12,5 & & 11,3 & & 16,4 & \\
\hline Medio & 36,7 & & 38,5 & & 34,2 & & 35,5 & & 31,9 & \\
\hline Medio-Bajo & 33,3 & & 32,4 & & 34,6 & & 35,8 & & 32,7 & \\
\hline Bajo & 7,6 & & 5,9 & & 11,2 & & 9,4 & & 10,6 & \\
\hline \multicolumn{11}{|l|}{ Funcionamiento familiar } \\
\hline Mayor funcionamiento & 63,6 & & 67,9 & & 51,4 & & 51,7 & & 55,8 & \\
\hline Menor funcionamiento & 36,4 & & 32,1 & & 48,6 & & 48,3 & & 44,2 & \\
\hline
\end{tabular}

$\mathrm{TPC}=$ total de problemas conductuales. $\mathrm{CI}=$ conductas intemalizantes. $\mathrm{CE}=$ conductas extemalizantes. 
La Tabla 2 muestra las frecuencias de los diagnósticos conductuales. Se observa que el $67,5 \%$ de la muestra es considerada normal; $69,6 \%$ en niñas y $65,4 \%$ en niños. El 21,4\%, 23,1\% y $16,4 \%$ tienen rango clínico en TPC, CI y CE respectivamente.

Las CI en rango alterado (borderline+clínico) fueron más frecuentes en niños con 36,1\% (IC $32,6-39,7 \%$ ) que en niñas 34\% (IC 30,4-37,6\%). Las CE en rango alterado fueron $30,6 \%$ (IC 27,2$34,0 \%$ ) en niños y $26,6 \%$ (IC 23,2-29,9\%) en niñas.

El 9,1\% (IC 7,5-10,7\%) de todos los cuidadores había tenido síntomas depresivos en los últimos 12 meses; en mujeres la frecuencia fue 9,2\% (IC 7,6-10,9\%) y en hombres 3,6\% (IC -3,3-10,4\%). La Tabla 3, muestra como hay una mayor frecuencia de cuidadores con síntomas depresivos a peor diagnóstico conductual.

El análisis bivariado muestra la relación entre problemas conductuales y las principales variables modificadoras de efecto. Destaca en el niño/a, asociaciones estadísticamente significativas con mayor número de enfermedades crónicas para las 3 escalas, la edad para CI y la asistencia a jardín infantil para TPC. En el cuidador destaca, el estar divorciado, tener eventos estresantes en el último tiempo, percibir la crianza de mucho esfuerzo emocional y tener síntomas depresivos para las 3 escalas, y desempleo para TPC, el sexo para CE y el nivel educacional para CI. A nivel familiar, destaca la asociación con disfunción familiar para las 3 escalas.

$\mathrm{Al}$ ingresar todas las variables significantes $\mathrm{y}$ otras teóricamente relevantes como el NSE, edad y nivel educacional del cuidador en un modelo de regresión logística multinomial, explican la presencia de problemas conductuales: número de enfermedades crónicas del niño/a, número de eventos vitales estresantes vividos en el último año, demanda emocional de mucho esfuerzo en la crianza y presencia de síntomas depresivos en el cuidador, y disfunción a nivel familiar (Tabla 4). Llama la atención que al ingresar al modelo, la significancia de la asociación entre síntomas depresivos y CI se pierde.

\section{Discusión}

Analizamos la asociación entre depresión del cuidador y problemas conductuales en el preescolar en el contexto de determinantes sociodemográficos, médicos y familiares.

Es conocida esta asociación, pero hay pocos estudios que exploren la potencia de ésta ante

Tabla 2. Frecuencia de problemas conductuales

\begin{tabular}{|lccccccccc|}
\hline $\begin{array}{l}\text { Diagnóstico } \\
\text { conductual }\end{array}$ & \multicolumn{3}{c}{ Diagnóstico TPC } & \multicolumn{3}{c}{ Diagnóstico CI } & \multicolumn{3}{c|}{ Diagnóstico CE } \\
Normal & $\mathbf{n}$ & $\mathbf{\%}$ & IC 95\% & $\mathbf{n}$ & $\%$ & IC 95\% & n & \% & IC 95\% \\
Borderline & 929 & 675 & $64,9-69,9$ & 894 & 649 & $62,3-67,4$ & 983 & 714 & $68,9-73-7$ \\
Rango clínico & 153 & 111 & $9,5-12,9$ & 165 & 120 & $10,3-13-8$ & 168 & 122 & $10,5-14,1$ \\
\hline
\end{tabular}

$\mathrm{TPC}=$ total de problemas conductuales. $\mathrm{CI}=$ conductas intemalizantes. $\mathrm{CE}=$ conductas extemalizantes.

Tabla 3. Distribución por frecuencia de síntomas depresivos según rango de problemas conductuales

\begin{tabular}{|lcccc|}
\hline Diagnóstico & \multicolumn{2}{c}{ Conductas internalizantes } & \multicolumn{2}{c|}{ Conductas externalizantes } \\
conductual & \% síntomas depresivos & IC 95\% & \% síntomas depresivos & IC 95\% \\
Normal & 7,2 & $4,7-9,8$ & 7,2 & $5,5-8,9$ \\
Borderline & 9,5 & $4,7-14,2$ & 11,5 & $6,5-16,6$ \\
Rango clínico & 14,5 & $10,3-18,6$ & 16,1 & $10,9-21,2$ \\
\hline
\end{tabular}


Tabla 4. Regresión logística multivariada de los problemas conductuales

\begin{tabular}{|c|c|c|c|c|c|c|}
\hline \multirow{2}{*}{$\begin{array}{l}\text { Características de los niños/as y sus cuidadores } \\
\text { Del niño/a }\end{array}$} & \multicolumn{2}{|c|}{ TPC $n=295$} & \multicolumn{2}{|c|}{$\mathrm{CI} n=318$} & \multicolumn{2}{|c|}{$\mathrm{CE} n=226$} \\
\hline & OR & IC 95\% & OR & IC 95\% & OR & IC 95\% \\
\hline $\mathrm{N}^{\circ}$ de enfermedades crónicas en la infancia & $1,27^{\star * *}$ & $1,14-1,42$ & $1,26^{\star * *}$ & $1,14-1,40$ & $1,20^{\star *}$ & $1,07-1,34$ \\
\hline \multicolumn{7}{|l|}{ Del cuidador principal } \\
\hline $\mathrm{N}^{\circ}$ de eventos vitales estresantes & $1,20^{\star *}$ & $1,05-1,36$ & $1,23^{\star \star *}$ & $1,09-1,39$ & 1,12 & $0,99-1,27$ \\
\hline \multicolumn{7}{|l|}{ Emocionalmente, atender al niño/a le demanda } \\
\hline Mucho esfuerzo & 1 & & 1 & & 1 & \\
\hline Moderado esfuerzo & $1,5^{*}$ & $1,05-2,06$ & 1,31 & $0,94-1,81$ & $1,97^{* * *}$ & $1,33-2,91$ \\
\hline Escaso esfuerzo & $2,74^{\star * *}$ & $1,84-4,09$ & $1,95^{\star * *}$ & $1,32-2,87$ & $4,63^{\star * *}$ & $3,01-7,13$ \\
\hline \multicolumn{7}{|l|}{ Síntomas depresivos del cuidador } \\
\hline No & 1 & & 1 & & 1 & \\
\hline Sí & $1,73^{*}$ & $1,09-2,76$ & 1,31 & $0,82-2,09$ & $1,7^{*}$ & $1,02-2,82$ \\
\hline \multicolumn{7}{|l|}{ Del grupo familiar } \\
\hline \multicolumn{7}{|l|}{ Funcionamiento familiar } \\
\hline Mayor funcionamiento & 1 & & 1 & & 1 & \\
\hline Menor funcionamiento & $1,5^{*}$ & $1,08-1,99$ & $1,68^{\star * *}$ & $1,25-2,26$ & 1,16 & $0,82-1,63$ \\
\hline
\end{tabular}

TPC $=$ total de problemas conductuales. $\mathrm{CI}=$ conductas intemalizantes. $\mathrm{CE}=$ conductas extemalizantes. ${ }^{\star} \mathrm{p}<0,05^{\star}$; ${ }^{* *} \mathrm{p}<0,01 ;{ }^{* *} \mathrm{p}<0,001.1=$ referencia.

posibles confundentes. En nuestro estudio, la depresión del cuidador principal se relacionó significativamente con niveles más altos de problemas conductuales. Esta asociación fue moderada por covariables teórica y metodológicamente relevantes, donde las enfermedades crónicas del niño, la demanda emocional de mucho esfuerzo que supone la crianza, los eventos vitales estresantes para el cuidador en el último año y el funcionamiento familiar, mantienen su significancia para TPC y CI. Para CE mantienen significancia las variables enfermedades crónicas y demanda emocional del cuidador y no las otras. Por otro lado, nuestra variable índice, síntomas depresivos, mantiene su asociación significativa con TPC y CE.

Conocer esto tiene relevancia en nuestro quehacer ya que nos obliga frente a una díada donde hay TPC y sospecha de síntomas depresivos, en un contexto social adverso (covariables significativas), a evaluar depresión en el cuidador, ya que la asociación es tan potente que por sí sola puede explicar el comportamiento del niño. Lo mismo ocurre para CE en un contexto donde predominen las enfermedades crónicas del niño y una alta demanda emocional en la crianza. En CI y síntomas depresivos del cuidador será importante ponderar hasta qué punto las covariables significativas en el modelo explican el comportamiento, fenómeno que ya han intentado explicar otros estudios ${ }^{24}$.

Las características tanto del niño como del entorno, independientemente y en interacción, parecen contribuir a la aparición de estas conduc$\operatorname{tas}^{25}$ desde temprana edad, dada la dependencia del entorno que en esta etapa es principalmente el familiar ${ }^{26}$ variando en severidad, duración y prevalencia ${ }^{27}$ según los distintos contextos que son objeto de este estudio.

Estos resultados muestran la complejidad del análisis del comportamiento del preescolar que, además, queda sujeto a patrones de estabilidad descritos antes de los 30 meses de vida, fuerte- 
mente asociados a la depresión materna o del cuidador (en este caso en su mayoría mujeres), pero también a factores intrínsecos del niño como el temperamento que a su vez interactúan bidireccionalmente, y que podrían explicar la variabilidad de los resultados ${ }^{28}$.

Es sabido que la salud mental infantil y los problemas conductuales presentan una clara gradiente socioeconómica $^{28}$. Del mismo modo, la baja escolaridad del cuidador y que a mayor nivel educacional mejoran los resultados conductuales ${ }^{29}$.

En nuestro estudio, el promedio de años de estudio fue menor que el promedio nacional (10,6 años, CASEN 2011) y sobre dos tercios de la muestra provienen de NSE medio y medio-bajo, lo que es concordante porque la muestra proviene del sistema de salud público. Aun así, se observó mayor prevalencia de TPC, CI y CE en NSE bajo.

Los bajos ingresos vinculados a problemas conductuales, estarían mediados por la respuesta al estrés que genera la pobreza, aumentando los niveles de conflicto y sobrecarga en la crianza ${ }^{30}$, el aumento de ingresos en NSE bajo se asocia a disminución de CE y CI entre los 2 y 5 años. Bajos ingresos con cuidadores de más nivel educacional, se asocian a menor puntuación en $\mathrm{CBCL}^{31,32}$.

El divorcio presenta significancia estadística en el análisis bivariado para las 3 escalas y el desempleo también para TPC, resultados concordantes con literatura internacional ${ }^{33,34}$.

Por otro lado, nos parece importante analizar los resultados obtenidos directamente de la aplicación del CBCL-11/2-5 donde obtuvimos un score total promedio para TPC de $43,21 \pm 28,6$, puntaje superior a los encontrados en estudios internacionale ${ }^{27,35}$ con una prevalencia en rango clínico de $21,4 \%$, similar a estudios en EE. UU. (14\%-26\%) ${ }^{1}$, pero distantes de estudios canadienses, holandeses y finlandeses $(11,1 \%, 7,8$ y $7,9 \%$ respectivamente) $)^{32,36,37}$.

Las prevalencias para CI y CE en rango clínico en nuestro estudio fueron $23,1 \%$ y $16,4 \%$ respectivamente. Si bien es mayor la prevalencia para las CI, su score total promedio fue más bajo $(12,31 \pm 10,56)$ en relación a CE $(15,44 \pm 9,27)$, con una razón $\mathrm{CE} / \mathrm{CI}=1,3$, resultados que son similares a los reportados por otros estudios chilenos y extranjeros ${ }^{38,39}$. Entre las posibles explicaciones para la alta prevalencia de CI en relación a CE se debe considerar un probable subreporte de CE de parte del cuidador, por considerarlas normales para la edad. Estudios sobre estabilidad de los patrones en el tiempo muestran que el patrón internalizante emerge hacia el final del período preescolar, mostrando una diferencia significativa respecto de menores de 3 años $^{28}$. Si bien en nuestro estudio la CI fue más prevalente, su intensidad es menor (score promedio total más bajo) que las $\mathrm{CE}$, fenómeno que sería interesante estudiar en nuestra cultura.

En relación a lo anterior, en preescolares asíaticos/chinos, es mayor la intensidad de problemas internalizantes que externalizantes; por el contrario en EE. UU. la situación es a la inversa ${ }^{35}$. Esto ha sido explicado por el modelo de supresión-facilitación de problemas, en que prácticas culturales favorecen la expresión de ciertos modos conductuales por sobre otros y a diferencias temperamentales. Por otra parte sabemos que, por ejemplo, en Chile la madre reporta significativamente más problemas que los profesores y principalmente internalizantes ${ }^{40}$.

Finalmente, para nosotros fue importante explorar la asociación entre los problemas conductuales y los síntomas depresivos del cuidador, y junto a ello, las posibles variables confundentes de la asociación no sólo porque permitieron depurar la asociación buscada, sino porque cada una de ellas, analizada independientemente de forma bivariada, nos permitió aproximarnos a la compresión del complejo fenómeno en el que se enmarca el desarrollo evolutivo del niño donde la dependencia del entorno próximo es vital.

En conclusión, podemos afirmar la asociación entre síntomas depresivos del cuidador principal y problemas conductuales en la población estudiada es significativa. Creemos que es esencial tener identificados los grupos de mayor riesgo para enfocar los posibles objetivos promocionales y preventivos en esta materia mediante acciones sobre los determinantes de la salud que inciden en la interacción diádica. 


\section{Resumen}

Introducción: La salud mental del cuidador influye en el desarrollo de los niños; sin embargo, desconocemos si existe una asociación entre sintomas depresivos del cuidador y problemas conductuales en preescolares chilenos. El objetivo fue analizar la asociación entre los sintomas depresivos del cuidador principal y la presencia de problemas conductuales en preescolares usuarios de la red pública de salud chilena. Método: Estudio transversal. Muestreo por conglomerados estratificados bietápico. Muestra de 1.377 preescolares de 2,5-4 años y sus cuidadores principales. Con consentimiento informado se aplicó encuesta sociodemográfica y Child Behavior Checklist 11/2-5. Los sintomas depresivos del cuidador se evaluaron mediante Entrevista Diagnóstica Internacional Compuesta abreviada. Resultados: Edad promedio del preescolar 40,95 \pm 4,6 meses, 51,3\% varones. 97,5\% fueron cuidadoras mujeres, edad promedio 31,6 $\pm 10,2$ años y escolaridad 9,5 años. Se encontró problemas conductuales de rango clínico en las tres escalas: total de problemas conductuales $21,4 \%$, conductas internalizantes $23,1 \%$ y externalizantes $16,4 \%$. Se encontró sintomatología depresiva en 9,1\% de los cuidadores, la que aumenta a 14,5,\% y 16,1\% en cuidadores de preescolares con conductas internalizantes y externalizantes respectivamente. En el análisis de regresión logística multinomial ajustando por variables confundentes, la asociación bivariada con los problemas conductuales internalizantes desaparece, sin embargo, se mantiene para el total de problemas conductuales y conductas externalizantes. Conclusiones: La asociación entre sintomas depresivos del cuidador y problemas conductuales en preescolares chilenos es consistente y modulada por otras variables médicas y psicosociales, esto sugiere la importancia de la identificación, prevención e intervención temprana de la díada y su entorno.

Palabras clave: Preescolar, Depresión del Cuidador, CBCL.

\section{Referencias bibliográficas}

1. Egger H, Angold A. Common emotional and behavioral disorders in preschool children: Presentation, nosology and epidemiology. J Child Psychol Psychiatry 2006; 47: 313-37.

2. Postert Ch, Averbeck-Holocher M, Beyer T, Müller J, Furniss T. Five systems of psychiatric classification for preschool children: do differences in validity, usefulness and reliability make for competitive or complimentary constellations? Child Psychiatry Hum Dev 2009; 40 (1): 25-41.

3. Achenbach T, Rescorla L. Manual for the ASEBA Preschool Forms \& Profiles. Burlington, VT: University of Vermont Department of Psychiatry, Research Center for Children, Youth, \& Families. 2000.

4. Achenbach T. Clinical models for child and ado- lescent behavioral, emotional and social problems in: JM. Rey. IACAPAP Textbook of Child and Adolescent Mental Health. Geneva. International Association for Child and Adolescent Psychiatry and Allied Professions; 2012. Section A.3: 1-20.

5. Briggs-Gowan $\mathrm{M}$, Bonsson-Heenan J, Carter A, Guyer A, Horwitz S. Are infant-toddler socialemotional and behavioral problems transient?. J Am Acad Child Adolesc Psychiatry 2006; 45 (7): 849-58.

6. Lavigne J, Arend R, Rosenbaum D, Binns H, Christoffel K, Gibbons R. Psychiatric disorders with onset in the preschool years: I. Stability of diagnoses. J Am Acad Child Adolesc Psychiatry 1998; 37: 1246-54.

7. Shaw D, Gilliom M, Ingoldsby E, Nagin D. Trajectories leading to school-age conduct problems. Dev Psychol 2003; 39 (2): 189-200. 
8. Essex M, Kraemer H, Slattery M, Burk, L, Boyce W, Woodward H, et al. Screening for childhood mental health problems: Outcomes and early identification. J Child Psychol Psychiatry 2009; 50 (5): 562-70.

9. Shaw D, Dishion T, Supplee L, Gardner F, Arnds K. Randomized trial of a family-centered approach to the prevention of early conduct problems: 2-year effects of the family check-up in early childhood. J Consult Clin Psychol 2006; 74 (1): 1-9.

10. Hill J. Biological, psychological and social processes in the conduct disorders. J Child Psychol Psychiatry 2002; 43 (1): 133-64.

11. Van Zeijl J, Mesman J, Stolk M, Alink L, Van Ijzendoorn M, Bakermans-Kranenburg $\mathrm{M}$, et al. Terrible ones? Assessment of externalizing behaviors in infancy with the child behavior checklist. J Child Psychol Psychiatry 2006; 47 (8): 801-10.

12. Zeanah C. Handbook of infant mental health (3rd ed.). 2009. New York: Guilford.

13. Putnam S, Rothbart M, Garstein M. Homotypic and heterotypic continuity of fine-grained temperament during infancy, toddlerhood, and early childhood. Infant and Child Development 2008; 17: 387-405.

14. Bagner D, Pettit J, Lewinsohn P, Seeley J. Effect of Maternal Depression on Child Behavior: A Sensitive Period? J Am Acad Child Adolesc Psychiatry 2010; 49 (7): 699-707.

15. Lovejoy M, Graczyk P, O’Hare E, Neuman G. Maternal depression and parenting behavior: a meta-analytic review. Clin Psychol Rev 2000; 20 (5): 561-92.

16. Weaver C, Shaw D, Dishion T, Wilson M. Parenting self-efficacy and problem behavior in children at high risk for early conduct problems: The mediating role of maternal depression. Infant Behav Dev 2008; 31 (4): 594-605.

17. Cummings E, Davies P. Maternal depression and child development. J Child Psychol Psychiatry 1994; 35 (1): 73-112.

18. Cummings E, Keller P, Davies P. Towards a family process model of maternal and paternal depressive symptoms: Exploring multiple relations with child and family functioning. J Child Psychol Psychiatry 2005; 46(5): 479-89.
19. Elgar FJ, McGrath PJ, Waschbusch DA, Stewart $\mathrm{SH}$, Curtis LJ. Mutual influences on maternal depression and child adjustment problems. Clin Psychol Rev 2004; 24 (4): 441-59.

20. Goodman S, Gotlib I. Risk for psychopathology in the children of depressed mothers: A developmental model for understanding mechanisms of transmission. Clin Psychol Rev 1999; 106: 458-90.

21. Seguel X, Edwards M, De Amesti A, Montenegro, H. Prevalencia de problemas conductuales y emocionales en la población preescolar de Santiago, 1992. Santiago: CEDEP.

22. Kessler R, Angermeyer M, Anthony J, Graaf $\mathrm{R}$, Demyttenaere K, Gasquet I, et al. Lifetime prevalence and age-of-onset distributions of mental disorders in the World Health Organization's World Mental Health Survey Initiative. World Psychiatry 2007; 6 (3): 168-76.

23. Lecannelier F, Pérez J, Groissman S, Gallardo D, Bardet AM, Bascuñán A, et al. Validation of Child Behavior Inventory for children ages $11 / 2$ to 5 years (CBCL 1 1/2-5) at the Santiago de Chile City. Universitas Psychologica 2014; 13: 491-500.

24. Campbell S, March C, Pierce E, Ewing L, Szumowski E. Hard-to-manage preschool boys: family context and the stability of externalizing behavior. J Abnorm Child Psychol 1991; 19 (3): 301-18.

25. Campbell S, Shaw D, Gilliom M. Early externalizing behavior problems: Toddlers and preschoolers at risk for later maladjustment. Dev Psychopathol. 2000 Summer; 12 (3): 467-88.

26. Lahey B, Van Hulle C, Keenan K, Rathouz $\mathrm{P}$, D’Onofrio B, Rodgers J, et al. Temperament and Parenting during the First Year of Life Predict Futurr Child Conduct Problems. J Abnorm Child Psychol 2008; 36 (8): 1139-58.

27. Kristensen S, Henriksen T, Bilenberg N. The Child Behavior Checklist for Ages 1.5-5 (CBCL/1(1/2)$5)$ : assessment and analysis of parent-and caregiver-reported problems in a population-based sample of Danish preschool children. Nord J Psychiatry 2010; 64 (3): 203-9.

28. Ringoot A, Van der Ende J, Jansen P, Measelle J, Basten M, So P, et al. Why mothers and young children agree or disagree in their reports of the 
child's problem behavior. Child Psychiatry Hum Dev 2015; 46 (6): 913-27.

29. Bedregal P, Hernández V, de la Cruz R, Valenzuela E, Prado P, Vergara V, et al. Levantamiento y análisis de información sobre desarrollo infantil y sus principales determinantes sociales y económicas, en el contexto del sistema Chile Crece Contigo. Ministerio de Desarrollo Social y Departamento de Salud Pública, Escuela de Medicina, Facultad de Medicina de la Pontificia Universidad Católica de Chile, sept 2010.

30. Heberle A, Krill S, Briggs-Gowan M, Carter A. Predicting externalizing and internalizing behavior in kindergarten: examining the buffering role of early social support. J Clin Child Adolesc Psychol 2015; 44 (4): 640-54.

31. Dearing E, McCartney K, Taylor B. Withinchild associations between family income and externalizing and internalizing problems. Dev Psychol 2006; 42 (2): 237-52.

32. Loeb S, Fuller B, Kagan S, Carrol B. Child care in poor communities: early learning effects of type, quality, and stability. Child Dev 2004; 75 (1): 47-65.

33. Koot H, Verhulst F. Prevalence of problem behavior in Dutch children aged 2-3. Acta Psychiatr Scand Suppl 1991; 367: 1-37.

34. Robbers S, van Oort F, Huizink A, Verhulst F, van Beijsterveldt C, Boomsma D, et al. Childhood problem behavior and parental divorce: evidence for gene-environment interaction. Soc Psychiatry
Psychiatr Epidemiol 2012; 47 (10): 1539-48.

35. Liu J, Cheng H, Leung P. The application of the preschool child behavior checklist and the caregiver-teacher report form to mainland chinese children: syndrome structure, gender differences, country effects, and inter-informant agreement. J Abnorm Child Psychol 2011; 39 (2): 251-64.

36. Larson C, Pless I, Miettinen O. Preschool behavior disorders: their prevalence in relation to determinants. J Pediatr 1988; 113(2): 278-85.

37. Sourander A. Emotional and behavioural problems in a sample of Finnish three-year-olds. Eur Child Adolesc Psychiatry 2001; 10 (2): 98-104.

38. Cova F, Bustos C, Streiner D, Rincón P, Saldivia S, Grandón P, et al. Inventario de Conductas Infantiles en preescolares: Propiedades psicométricas del Inventario de Conductas Infantiles (CBCL/1.5-5) y del Informe del Cuidador/Educador (C-TRF) en Preescolares Chilenos. Terapia psicológica, 2016; 34 (3): 191-8.

39. Rescorla L, Achenbach T, Ivanova M, Harder V, Otten L, Bilenberg N, et al. International comparisons of behavioral and emotional problems in preschool children: parents' reports from 24 societies. J Clin Child Adolesc Psychol 2011; 40 (3): 456-67.

40. Gómez A, Santelices M. Problemas conductuales en preescolares chilenos: Percepción de las madres y del personal educativo. Estudios Pedagógicos XL. 2014; (2): 175-87.

\footnotetext{
Correspondencia:

Dra. Paula Bedregal

Departamento de Salud Pública, Pontificia Universidad Católica de Chile.

Diagonal Paraguay \#362, $2^{\circ}$ piso.

Código Postal 8330077.

Teléfono: +56223533038 .

pbedrega@gmail.com.
} 